\title{
The effect of $\mathrm{A} \beta$ and PKC on actin network remodelling
}

\author{
Henriques, A. G., Carvalho, L. P., Oliveira, J. M. and da Cruz e Silva, O. A. B.
}

Laboratório de Neurociências, Centro de Biologia Celular, Secção Autónoma de Ciências da Saúde, Universidade de Aveiro, Campus de Santiago, 3810-193 Aveiro, Portugal

Email: odetecs@ua.pt

Cytoskeletal abnormalities have been reported in Alzheimer's disease (AD), some of which mediated by the neurotoxic peptide $A \beta$, including increase in F-actin polymerization. A $\beta$ results from the proteolytic processing of its precursor, the Alzheimer's Amyloid Precursor Protein (APP), being that aggregation of the former is a key event in AD. By confocal microscopic analyses we previously show that $\mathrm{A} \beta$ incubation for $24 \mathrm{hr}$ leads to rearrangements in both actin and tubulin networks [1,2]. Here we address the molecular basis of the actin cytoskeleton rearrangements. It is well known that cytoskeleton dynamics can be regulated by intracellular signals, which can also be modulated by exogenous stimulus. Protein kinase C (PKC) is one of the molecules involved in the regulation of cellular cytoskeleton-driven processes [3], and the activity of the latter kinase can be affected by $\mathrm{A} \beta$ peptide [4].

This work addressed the effects of $\mathrm{A} \beta$ and PKC on actin network remodelling. In agreement with our previous work, A $\beta$-treated HeLa cells showed an increase in the F-actin, mainly arranged as stress fibers, when compared to untreated cells. Interestingly, treatment with the PKC stimulator, PMA (phorbol-12myristate-13-acetate) at $0.5 \mu \mathrm{M}$, results in an increase in the filamentous actin network (small fibrils) and punctuated F-actin, a strikingly different phenotype from that observed for $\mathrm{A} \beta$ alone. Co-incubation with both $\mathrm{A} \beta$ and PMA gives an actin phenotype close to that observed for PMA alone, although in some cells the filaments formed appear to be longer. Besides stimulating PKC, PMA can also affect other cellular processes and the same is true for $\mathrm{A} \beta$, which can affect both PKC [4] and protein phosphatase activities [5]. Hence, different modes of action may underlie the different actin phenotypes observed. Additionally, PKC was inhibited using rottlerin. Rottlerin alone, at concentrations reported to inhibit PKC $\delta$ produced a dramatic remodeling of the actin cytoskeleton. Under these conditions, filamentous structures, consistent with stress fibers were observed in a subpopulation of cells. Further, punctate F-actin structures were evident throughout the cytoplasm, but the most notorious change were the pronounced thin filopodia-like structures that extend out from the cells. Inhibition of the different PKC isoforms led to remodeling of the cytoskeleton with distinct F-actin morphological phenotypes (data not shown), but additional studies are needed to clarify the different PKC isoforms mediated effects on the actin network. Nonetheless, inhibition of PKC at $10 \mu \mathrm{M}$ rottlerin partially reverts the $\mathrm{A} \beta$ effect, as denoted by co-incubation experiments. Taken together the data support the notion that $A \beta$ effects on actin network involves at least, in part, PKC activity modulation. Of note, actin dynamics can involve the crosstalk between kinases and phosphatases and consequently the target of different or juxtaposing signaling pathways (as the direct phosphorylation and/or dephosphorylation of cytoskeletal regulators) that will impact on actin cytoskeleton. Hence, the comprehension of the signalling events involved in actin dynamics will improve our knowledge on the molecular basis underlying cytoskeleton network remodeling in AD pathology.

Acknowledgements: This work was supported by the Fundação para a Ciência e Tecnologia of the Portuguese Ministry of Science and Technology: PTDC/BEX-BCM/0493/2012, PTDC/QUIBIQ/101317/2008 and JPND BIOMARKAPD - Biomarkers for Alzheimer's disease and Parkinson's disease', and also the Center for Cell Biology UA - REEQ/1023/BIO/2005. 
[1] Henriques et al., Microscopy and Microanalysys, 18, 23-24, 2012

[2] Henriques et al., Journal of Neurochemistry, 113, 761-771, 2010

[3] Larsson, Cellular Signalling. 18, 276-284, 2006

[4] Kuperstein et al., Journal of Neurochemistry 76, 758-767, 2001

[5] Vintém et al., Neurotoxicology and Terantology, 31, 85-88, 2009

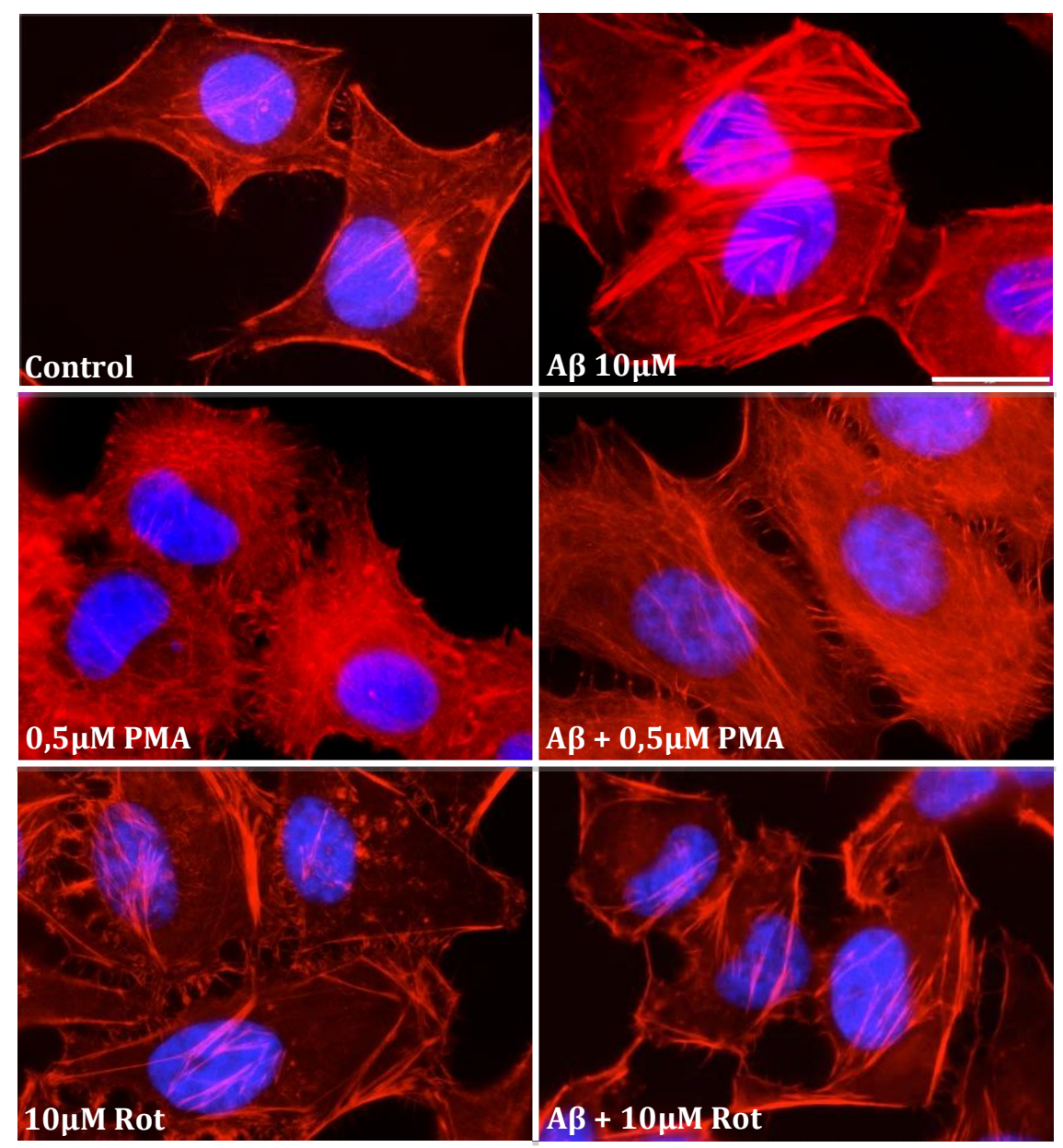

Figure 1. Involvement of $A \beta$ and PKC activity modulation in actin cytoskeleton rearrangements.

HeLa cells were treated with $A \beta_{25-35}(10 \mu \mathrm{M}, 24 \mathrm{hr})$. To address PKC involvement on actin remodeling cells were incubated with PMA (phorbol-12-myristate-13-acetate; PKC stimulator) or with rotlerin (PKC inhibitor) during 1hr, or co-incubated with $A \beta_{25-35}$, where drugs were added in the last hr of incubation period with $A \beta$. HeLa cells were then stained with Alexa Fluor 568 phalloidin (F-actin staining, red) and DAPI (nuclear staining, blue). Epifluorescence images were acquired with an Olympus IX-81 motorized inverted epifluorescence microscope equipped with the appropriate filter combinations and a 100×objective (Plan-Neofluar, 100×/1.35 oil objective). Scale bar, $20 \mu \mathrm{m}$. 